\title{
RESEARCH
}

Open Access

\section{Exosomal microRNA-22-3p alleviates cerebral ischemic injury by modulating $\mathrm{KDM} 6 \mathrm{~B} / \mathrm{BMP} 2 / \mathrm{BMF}$ axis}

\author{
Yamei Zhang ${ }^{1 * \dagger} \mathbb{D}$, Junying $\mathrm{Liu}^{1+}, \mathrm{Mi} \mathrm{Su}^{1}$, Xin Wang ${ }^{1}$ and Chenchen $\mathrm{Xie}^{2}$
}

\begin{abstract}
Background: Cerebral ischemia-reperfusion (I/R) injury, the most common form of stroke, has high mortality and often brings persistent and serious brain dysfunction among survivors. Administration of adipose-derived mesenchymal stem cells (ASCs) has been suggested to alleviate the I/R brain injury, but the mechanism remains uncharacterized. Here, we aimed at investigating the mechanism of ASCs and their extracellular vesicles (EVs) in the repair of or protection from I/R injury.
\end{abstract}

Methods: We established the middle cerebral artery occlusion (MCAO) model and oxygen-glucose deprivation/ reperfusion (OGD/RP) neuron model. ASCs or ASC-derived EVs (ASC-EVs) were co-cultured with neurons. RT-qPCR and Western blot analyses determined microRNA (miRNA)-22-3p, BMP2, BMF, and KDM6B expression in neurons upon treatment with ASC-EVs. Bioinformatics analysis predicted the binding between miR-22-3p and KDM6B. Using gain- and loss-of-function methods, we tested the impact of these molecules on I/R injury in vivo and in vitro.

Results: Treatment with ASCs and ASC-derived EVs significantly alleviated the I/R brain injury in vivo, elevated neuron viability in vitro, and decreased apoptosis. Interestingly, miR-22-3p was upregulated in ASC-EVs, and treatment with EV-miR-22-3p inhibitor led to increased apoptosis and decreased neuronal. Of note, miR-22-3p bound to and inhibited KDM6B, as demonstrated by dual-luciferase reporter gene assay and Western blot assay. Overexpression of KDM6B enhanced apoptosis of neurons in the OGD/RP model, and KDM6B bound to BMB2 and promoted its expression by binding to BMP2. Silencing of BMF reduced infarct volume and apoptosis in the stroke model.

Conclusion: Results support a conclusion that ASC-EV-derived miR-22-3p could alleviate brain ischemic injury by inhibiting KDM6B-mediated effects on the BMP2/BMF axis. These findings compelling indicate a novel treatment strategy for cerebral ischemic injury.

Keywords: Adipose-derived mesenchymal stem cells, Ischemia-reperfusion, Extracellular vesicles, microRNA-22-3p, KDM6B, BMP2, BMF

\footnotetext{
* Correspondence: yameizhang@126.com

†Yamei Zhang and Junying Liu contributed equally to this work.

${ }^{1}$ Clinical Genetics Laboratory, Affiliated Hospital \& Clinical Medical College of

Chengdu University, No. 82 North Second Section of Second Ring Road,

Jinniu District, Chengdu 610081, Sichuan Province, People's Republic of

China

Full list of author information is available at the end of the article
}

(C) The Author(s). 2021 Open Access This article is licensed under a Creative Commons Attribution 4.0 International License, which permits use, sharing, adaptation, distribution and reproduction in any medium or format, as long as you give appropriate credit to the original author(s) and the source, provide a link to the Creative Commons licence, and indicate if changes were made. The images or other third party material in this article are included in the article's Creative Commons licence, unless indicated otherwise in a credit line to the material. If material is not included in the article's Creative Commons licence and your intended use is not permitted by statutory regulation or exceeds the permitted use, you will need to obtain permission directly from the copyright holder. To view a copy of this licence, visit http://creativecommons.org/licenses/by/4.0/ The Creative Commons Public Domain Dedication waiver (http://creativecommons.org/publicdomain/zero/1.0/) applies to the data made available in this article, unless otherwise stated in a credit line to the data. 


\section{Background}

Stroke, the second-most cause of death worldwide, encompasses hemorrhagic stroke, but the majority of cases are caused by arterial occlusion causing ischemic injury [1]. Stroke results in acute neuronal cell death and focal brain inflammation, which aggravates secondary brain injury by exacerbating blood-brain barrier damage, microvascular failure, brain edema, and oxidative stress [2]. Thanks to technological innovations, the clinical management of ischemic stroke has greatly advanced, notably through the use of intravenous thrombolysis and endovascular thrombectomy, which reduces disability [3]. However, identification of pathways and molecules that participate in cerebral ischemia could reveal novel approaches to improve the clinical outcome [4]. In this regard, animal models mimicking human stroke such as the middle cerebral artery occlusion (MCAO) model enable the study on the pathogenesis of cerebral ischemia [5].

Mesenchymal stem cell (MSC) transplantation has been demonstrated to improve functional and pathological recovery in cerebral ischemia [6]. In the condition of cerebral ischemia, the MSC transplantation engenders the migration of endogenous neuronal progenitor cells and alleviates the neuroinflammation associated with acquired brain injury $[7,8]$. Of note, administration of adipose tissue-derived MSCs (ASCs) leads to functional recovery of cerebral lesions and reduces apoptosis, yet promotes neurogenesis and proliferation [9]. Besides, treatment with ASCs could suppress inflammatory factors, decrease neurological severity, and reduce the brain infarction volume [10]. However, the mechanism by which ASCs alleviate cerebral ischemic injury remains uncharacterized, thus calling for further studies.

Extracellular vesicles (EVs), which originate from the endosomal system, are involved in multiple physiological and pathological processes [11]. EVs from MSCs (MSCEVs) are highlighted to protect the brain from hypoxiaischemia by suppressing cerebral inflammation [12]. Furthermore, MSC-EVs attenuate postischemic immunosuppression in the peripheral blood after ischemia, thus improving neurological impairment [13]. The use of MSC-EV, as an alternative to MSCs, confers several advantages such as a higher safety profile, lower immunogenicity, and the ability of the cells to cross biological barriers [14]. Mounting evidence demonstrates that EVs from ASCs (ASC-EVs) regulate immune responses and delay the progression of diseases as diverse as experimental autoimmune encephalomyelitis and breast cancer [15-17]. ASC-EVs are also indicated to suppress neuronal apoptosis and activate autophagy, thereby ameliorating cerebral ischemia-reperfusion (I/R) injury in the rat MACO model [18]. microRNAs (miRNAs), which are key components of EVs, contribute to cell-to-cell communication both locally and systemically, directly affecting the process of osteogenic and adipogenic differentiation of MSCs $[19,20]$. For example, miRNA-22-3p was highlighted to exhibit a protective effect against cerebral I/R injury, where its overexpression inhibited inflammatory cytokines and the pro-apoptosis gene Bax [21]. Besides, miRNA-22-3p enhances the intrinsic regenerative abilities of primary sensory neurons [22]. Nevertheless, little is known about the role of miR-22-3p in cerebral ischemic injury and its interaction with EVs or ASCs. The KDM6B histone demethylase is noted to play a critical role in osteogenic commitment of MSCs by removing $\mathrm{H} 3 \mathrm{~K} 9 \mathrm{me} 3$ and $\mathrm{H} 3 \mathrm{~K} 27 \mathrm{me} 3$ [23]. Indeed, KDM6B is an epigenetic regulator that mediates transcriptional activation during differentiation of hematopoietic stem and progenitor cells as well as immune responses [24]. In one study, loss of KDM6B activity leads to depletion of phenotypic and functional hematopoietic stem cells, indicating that KDM6B is a key to stem cell self-renewal in response to inflammatory and proliferative stress [25]. JMJD3 (KDM6B) knockdown has been reported to improve neurological deficits and reduced infarct volume following ischemic injury [26]. Given this background, in the present study, we aimed to explore the mechanism by which miR-22$3 p$ and ASC-EVs in concert with KDM6B functioned in cerebral ischemic injury, using the MACO and oxygen and glucose deprivation (OGD) models. Interestingly, our results suggested that ASC-EVs protect brain injury through transferring miR-22-3p and that miR-22-3p inhibits histone demethylase KDM6B to exert protective activity.

\section{Methods}

\section{Ethical statement}

All animal experiments were performed with the approval of the Animal Ethics Committee of the Affiliated Hospital of Chengdu University. The experiments involving animals followed the recommendations in the Guide for the Care and Use of Laboratory Animals of the National Institutes of Health.

\section{Animal model}

Adult Sprague Dawley (SD) rats (male, 10-12 weeks, 250-300 g) purchased from the Hunan SJA Laboratory Animal Co., Ltd. (Hunan, China) were subjected to temporary focal MCAO to induce focal ischemia [18]. In brief, rats were fixed on a stainless steel operating table under anesthesia with $30 \mathrm{mg} / \mathrm{kg}$ sodium pentobarbital (Sigma-Aldrich, St. Louis, MO, USA). The rat left internal carotid was exposed, and a nylon suture was inserted and advanced through the carotid bifurcation until the origin of the middle cerebral artery was blocked. After occlusion for $1 \mathrm{~h}$, the suture was removed, 
followed by reperfusion. The sham-operated rats were subjected to the same surgery except without MCAO. Before the surgery, EVs $(100 \mu \mathrm{g} / \mathrm{kg} /$ day $)$ and siRNA (5 $\mathrm{nmol} / 20 \mathrm{~g} /$ day) were injected via the lateral cerebral ventricle daily, and again for three consecutive 3 days. Three days after reperfusion, the rats were euthanized for the following experiments.

\section{Isolation of ASCs}

After surgery, an adipose tissue sample was collected from normal SD rats and was treated as previously described [27]. In brief, the adipose tissue was minced and digested with $0.1 \%$ collagenase A (Roche Diagnostics, Mannheim, Germany) in PBS containing $1 \%$ bovine serum albumin (BSA; Roche Diagnostics). After Ficoll density centrifugation (Lymphoprep; Axis-Shield, Oslo, Norway), the cells were seeded $\left(100,000\right.$ cells $\left./ \mathrm{cm}^{2}\right)$ into wells and cleaned of unattached cells by changing the medium. The ASCs were cultured in Dulbecco's modified Eagle medium (DMEM) containing 10\% fetal bovine serum (FBS; 30067334, Thermo Scientific, Waltham, MA, USA). Flow cytometry was performed to assess the expression of ASC surface marker. In brief, ASCs were first digested by trypsin with the addition of $10 \%$ goat serum (16210064, Thermo Scientific, Waltham, MA, USA) to prevent unspecific binding. Then, the ASCs were incubated with fluorescein isothiocyanate (FITC)labeled primary monoclonal antibodies against CD73, CD44, CD90, CD34, HLA-DR, CD45, and CD166 (1:100, BioLegend, San Diego, CA, USA), with FITC-IgG as a control. Finally, the cells were washed with PBS, resuspended in goat serum, and analyzed by CyAn ADP Analyzer (Beckman Coulter, Brea, CA, USA).

\section{Phenotypic characterization of cultured ASCs}

ASCs were placed in the DMEM medium with a specific solution [28] for 21-28 days with changing of the medium every 2 days. Then, the cells were fixed with $4 \%$ paraformaldehyde and washed with $\mathrm{PBS}$, followed by examination for osteogenic, adipogenic, and chondrogenic differentiation potential by staining with Alizarin Red (2\%), Alizarin Blue (1\%), and Oil Red, respectively. Finally, the ASCs were observed and counted under an optical microscope (DM400, Leica, Wetzlar, German).

\section{Isolation of ASC-EVs}

FBS for culturing ASCs was ultra-centrifuged at $1 \times 10^{6} \mathrm{~g}$ (Beckman Coulter Avanti J-30I, USA) for $16 \mathrm{~h}$ to obtain EV-free FBS, which was applied to the following experiment to avoid contamination from EVs. ASCs (approximately $3.2 \times 10^{7}$ cells) at passages $2-3$ were cultured in DMEM. When the confluence reached $70 \%$, ASCs were continuously cultured in DMEM (with EV-free FBS) for another $24-48 \mathrm{~h}$, and then the medium was collected and ultra-centrifuged to isolate ASC-EVs as previously described [29]. ASC-conditioned medium (CM) was centrifuged twice at $500 \times g$ for $10 \mathrm{~min}$, twice at $2000 \times g$ for $15 \mathrm{~min}$, and twice at $10,000 \times g$ for $30 \mathrm{~min}$. The final supernatant was centrifuged again at $70,000 \times g$ for $1 \mathrm{~h}$ and washed with PBS to remove debris and large vesicles. The pellet was resuspended in $100 \mu \mathrm{L}$ of PBS and stored at $-80^{\circ} \mathrm{C}$.

\section{Characterization of EVs}

Analysis of the absolute size distribution of ASC-EVs was determined by dynamic light scattering (DLS) using Nanosizer $^{\mathrm{TM}}$ instrument (Malvern Instruments, Malvern, UK). EVs were diluted in $1 \mathrm{~mL}$ of PBS and injected into the NanoSight NS300 instrument. Particles were automatically tracked and sized based on Brownian motion and the diffusion coefficient. Besides, the EVs were observed under a transmission electron microscope (Hitachi H7500 TEM, Hitachi, Tokyo, Japan) after staining with $1 \%$ uranyl acetate. Western blot analysis was further performed to determine the specific surface marker of EV expression, including rabbit antibodies against CD63 (ab216130; 1:2000, Abcam, UK), TSG101 (ab125011; 1:10,000, Abcam), and Calnexin (ab92573; 1: 100,000, Abcam).

\section{Primary cortical neuron culture}

As previously described [30], rat primary cortical neurons were obtained from newborn rats. In brief, cerebral tissues were minced and digested in trypsin for $30 \mathrm{~min}$ followed by the cell suspension being centrifuged at $3000 \times g$ for $10 \mathrm{~min}$. The precipitate was resuspended in DMEM/F12 medium to adjust the cell concentration to $1 \times 10^{6} / \mathrm{mL}$. The cells were seeded into 96 -well plates coated with $10 \mathrm{mg} / \mathrm{L}$ poly-L-lysine (Sigma, St. Louis, MO, USA) and incubated for $72 \mathrm{~h}$. The cells then were incubated with arabinosylcytosine (Shanghai Yuanye Biotechnology Co., Ltd., Shanghai, China) in the medium for $24 \mathrm{~h}$ to suppress non-neuronal cell growth. Then, the cells were transferred to a normal medium, which was refreshed every $72 \mathrm{~h}$. Immunofluorescence was performed to determine the expression of rabbit anti-MAP2 (A17409, 1:200, ABclonal, Boston, USA) and thus identify neurons. Oxygen-glucose deprivation/reperfusion (OGD/RP) was performed as described previously [21]. In brief, cortical neurons were treated with glucose-free Earl's solution in $5 \% \mathrm{CO}_{2}$ and $95 \% \mathrm{~N}_{2}$. At $72 \mathrm{~h}$ after treatment, cells were subjected to OGD/RP.

\section{EV internalization}

Purified EVs were labeled with the Dil (Sigma-Aldrich). Neurons were seeded into 8-well chamber slides (Thermo Scientific ${ }^{\mathrm{TM}}$ ) at a density of $8 \times 10^{3}$ cells/well and incubated with $5 \mu \mathrm{L}$ of PKH67 for $24 \mathrm{~h}$ to allow 
internalization. After being washed with PBS twice, the neurons were fixed with $4 \%$ paraformaldehyde for 15 min, followed by staining with DAPI $(0.5 \mathrm{mg} / \mathrm{mL}$; Invitrogen, USA). Finally, the cells were photographed under a confocal microscope (Zeiss LSM 780; Zeiss, Jena, German).

\section{Infarct volume measurement}

The brain was removed and sliced into six sequential coronal sections $( \pm 5 \mathrm{~mm}, \pm 3 \mathrm{~mm}$, and $\pm 1 \mathrm{~mm}$ from the bregma). The sections were stained with $2 \% 2,3,5-$ triphenyltetrazolium chloride (TTC; Sigma) and fixed in $4 \%$ paraformaldehyde followed by photography with a digital camera (Kodak DC240, East-man Kodak Co., Ltd). Infarct volume was calculated according to the formula: lesion area of each section $=$ (contralateral hemisphere area/ipsilateral hemisphere area) $\times$ ipsilateral lesion area. The volume of the lesion is estimated by multiplying the total areas of the lesion by the thickness of the slices [31].

\section{Neuronal nuclei (NeuN) immunofluorescence}

The rat hippocampus section was pre-incubated with $0.3 \%$ Triton X-100 in PBS for 10 min and blocked with $0.1 \%$ Triton $\mathrm{X}-100$ for $1 \mathrm{~h}$. Primary rabbit anti-NeuN (1: 500, ab177487, Abcam) and secondary goat anti-rabbit immunoglobulin G (IgG; 1:100, AS011, ABclonal, USA) were employed for immunofluorescence. The samples were placed in a DAPI matrix for nuclear staining and examined by inverted microscopy (Olympus IX71, Tokyo, China) in five randomly selected fields of the hippocampus CA1 region. Positive NeuN cells were counted by ImageJ software.

\section{Terminal deoxynucleotidyl transferase-mediated dUTP nick end-labeling (TUNEL) staining}

TUNEL staining was performed to assess hippocampus apoptosis using the One-step TUNEL Apoptosis Assay kit (Green fluorescence) (Beyotime Institute of Biotechnology, Shanghai, China). In brief, $72 \mathrm{~h}$ after the establishment of the MCAO model, frozen sections of hippocampal tissue specimens were obtained. The sections were first fixed with $4 \%$ paraformaldehyde or Immunol Staining Fix Solution (P0098, Beyotime) for 30-60 min, followed by washing in PBS twice for 10 min. Sections were next incubated with PBS containing $0.5 \%$ Triton X-100 for $5 \mathrm{~min}$ and washed in PBS or HBSS twice. A total of $50 \mu \mathrm{L}$ of TUNEL solution was added to the sections for 60-min incubation in the dark. After sealing the sections with anti-fluorescence quenching solution, the sections were observed under a fluorescence microscope.

\section{Cell transfection}

Lentivirus vector pLVX-miR-22-3p mimic/inhibitor (Ambion, Carlsbad, CA); lentivirus packages overexpressed plasmids, OE-KDM6B, and OE-Bone morphogenetic protein 2 (BMP2) (GeneChem, Shanghai, China); and lentivirus-siRNA, si-KDM6B, and si-Bcl-2 modifying factor (BMF) (Guangzhou RiboBio Co., Ltd., Guangdong, China) were employed to treat the neurons. The neurons were then co-localized with ASC-EVs or CM or underwent OGD/RP. Moreover, in vivo siRNA (siBMF) experiments were performed with modified in vivo siRNA (Ribobio Guangzhou, China).

\section{Cell counting kit-8 assay (CCK-8) assay}

The neurons were seeded into a 96-well plate at a density of $1 \times 10^{4}$ cells/well. Optical density (OD) was measured at $450 \mathrm{~nm}$ using a microplate reader and CCK-8 kit (Dojindo Laboratories, Kumamoto, Japan). Cell viability was calculated as the following formula: viability $(\%)=$ experiment group $(\mathrm{OD}) / \mathrm{NC}$ group $(\mathrm{OD}) \times 100 \%$.

\section{Dual-luciferase reporter gene assay}

pGL3 enhancer vector (Genscript, Nanjing, China) was cloned into rat KDM6B-wild-type (WT)-3'untranslated region (3'UTR) or KDM6B-mutant (MUT)-3'UTR, where the binding site of miR-22-3p was included. HEK293T cells (from American Type Culture Center) were cultured in 24-well plates for $24 \mathrm{~h}$ and then cotransfected with luciferase reporter vector miR-22-3p mimic or NC mimic according to the instructions of $\mathrm{Li}$ pofectamine 3000 Reagent (Invitrogen, USA). After $48 \mathrm{~h}$, the luciferase activity was detected by Dual-Luciferase Reporter Assay System.

\section{Chromatin immunoprecipitation (ChIP)}

ChIP was conducted to quantify the enrichment of KDM6B and H3K27me3 in the BMP2 promotor region, using the detection kit (Millipore corp., Billerica, MA, USA). Specifically, the cells were crosslinked with $1 \%$ formaldehyde and then resuspended in SDS lysis buffer. After sonicating to disrupt the nucleus, protein A/Gbeads was added to remove chromatin components. Then, anti-H3K27me3 antibody and anti-KDM6B antibody (ab38113, ABCAM) was added for incubation, with anti-rabbit IgG (ab171870, ABCAM), or anti-mouse IgG (ab81032, ABCAM) as NC. Finally, after decrosslinking and Proteinase $\mathrm{K}$ digestion, we used RT-qPCR to amplify and quantify the ChIP DNA, or IgG in the control samples. The primers for BMP2 were as follows: forward, 5'-CGTCTAGTATTTTGGCATAGCATAGACG-3'; reverse, 5' -ACTCAATTTCCAGCCTGCTGTTT-3'. 


\section{Reverse transcription quantitative polymerase chain reaction ( $R T-q P C R$ )}

Total RNA was extracted using Trizol Reagent (Invitrogen, Car, CA, USA) and reversely transcribed into cDNA with Revert Aid first-strand cDNA synthesis kit (Fermentas, Life Sciences, Canada). We utilized the SeraMir Exosome RNA Purification Kit (System Biosciences, Mountain View, USA) to isolate EV-miRNA. The synthesized cDNA was subjected to RT-qPCR based on the specifications of Fast Universal SYBR Green Master Mix (Roche, Indianapolis, USA) and ABI PRISM ${ }^{\bullet} 7900 \mathrm{HT}$ System (Takara Biotechnology, Japan), with the miRNAspecific forward primer (Sangon Biotech, Shanghai, China) and reverse primer from TaqMan microRNA assay kit (Table 1). In addition, we draw standard curves $\left(2^{-\triangle \Delta C T}\right)$ with GADPH as an internal reference. miR-22$3 \mathrm{p}$ in culture medium and EVs was normalized against the exogenous reference Cel-miR-39.

\section{Western blot analysis}

Protein was separated using sodium dodecyl sulfatepolyacrylamide gel electrophoresis (SDS-PAGE) and transferred to polyvinylidene fluoride membranes (Immobilon P, Millipore, Billerica, USA), which were blocked in Tris-buffered saline containing milk (5\%) and Tween-20 (0.1\%) at room temperature for $1 \mathrm{~h}$, followed by incubation with primary antibodies and horseradish peroxidase-labeled secondary antibody. Primary antibodies used in the experiment from ABclonal Biotechnology Co., Ltd. (Boston, USA) included rabbit antibodies against KDM6B (A17382, 1:2000), BMP2 (A5796, 1:2000), BMF (A5796, 1:2000), caspase 3 (A2156, 1:2000), cleaved caspase 3 (ab32042, 1:1500), Bax (A0207, 1:2000) and Bcl-2 (A0208, 1:2000), horseradish peroxidase-conjugated anti-rabbit IgG (AS014, 1: 10,000), and anti-mouse IgG (AS003, 1:10,000). The

Table 1 Primer sequences for PCR

\begin{tabular}{ll}
\hline Genes & Sequence \\
\hline miR-22-3p & F: 5'-GTGAAGCTGCCAGTTGAAG-3' \\
& R: 5'-GTGCAGGGTCCGAGGT-3' \\
Cel-miR-39-3p & 5'-UCACCGGGUGUAAAUCAGCUUG-3' \\
& R: 5'-A ACGCTTCACG A ATTTGCGT-3' \\
KDM6B & F: 5'-CAACTCCATCTGGCTGTTACTG-3' \\
& R: 5'-CCTTCTGCAACCAATTCCAG-3' \\
GAPDH & F: 5'-AGGTCGGTGTGAACGGATTG-3' \\
& R: 5'-GGGGTCGTTGATGGCAACA-3' \\
BMP2 & F: 5'-GGGACCCGCTGTCTTCTAGT-3' \\
& R: 5'-TCAACTCAAATTCGCTGAGGAC-3' \\
BMF & F: 5'-GGAGCGGGCGTATTTGGAA-3' \\
& R: 5'-ACACTCGATTGGGAAGAAGGG-3' \\
\hline
\end{tabular}

$F$ forward, $R$ reverse bands were visualized using enhanced chemiluminescence reagents (Thermo Fisher Scientific, Waltham, USA) and images were taken by ChemiDoc XRS Plus luminescent image analyzer (Bio-Rad, CA, USA). ImagePro Plus 6.0 software was applied to quantify band intensity, and the intensity of mouse anti-GAPDH (AC033, 1:50,000, ABclonal, USA) was applied to standardize target protein expression.

\section{Statistical analysis}

The data were processed using SPSS 21.0 statistical software (SPSS Statistics, Chicago, IL, USA). Measurement data were presented as mean \pm standard deviation. The data between two groups was analyzed by an independent $t$ test. The data among multiple groups were analyzed by one-way analysis of variance (ANOVA) with Tukey's post hoc test. ${ }^{*} p<0.05$ was considered statistically significant.

\section{Results}

ASC-EVs are protective towards neurons under cerebral ischemic injury

ASCs have been reported to attenuate brain ischemic injury [32]. To further explore the involved mechanism, we first isolated ASCs and performed flow cytometry to identify classic MSC surface marker expression. We found highly expressed CD73 (99\%), CD44 (96\%), CD90 (97\%), and CD166 (87\%) as well as lowly expressed CD34 (5\%), HLA-DR (0.5\%), and CD45 (0.2\%). Besides, the cells exhibited capacities of osteogenesis, adipogenesis, and chondrogenesis (Fig. 1a), confirming the effective isolation of ASCs. Meanwhile, rat primary cortical neurons were extracted successfully with purity over $95 \%$, as identified by immunofluorescence (Fig. 1b). Next, the neurons incubated in CM containing ASCs or EV-free CM were subjected to OGD/RP, which decreased dramatically the neuron viability, which was rescued by the addition of CM (Fig. 1c). However, depletion of EVs in CM led to even lower viability. Next, neurons were indirectly co-cultured with ASCs or ASCs treated with GW4869, an inhibitor of exosome biogenesis/release, and subjected to GOD/RP treatment. This showed that the promoting effect of ASCs on neuron viability was inhibited by GW4869 (Fig. 1d). Collectively, ASCs could alleviate the neuronal response to the injury induced by OGD/RP, wherein EVs of ASCs played a key role.

To verify the protective effect of EVs, ASC-EVs were isolated and characterized. EVs were spherical and cupshaped, with a diameter distribution around $100 \mathrm{~nm}$ (Fig. 1e, f). Western blot analysis further determined that the expression of CD63 and TSG101 increased in EVs (Fig. 1g), suggesting that EVs had been successfully isolated. Besides, with EVs labeled with Dil and neuron 


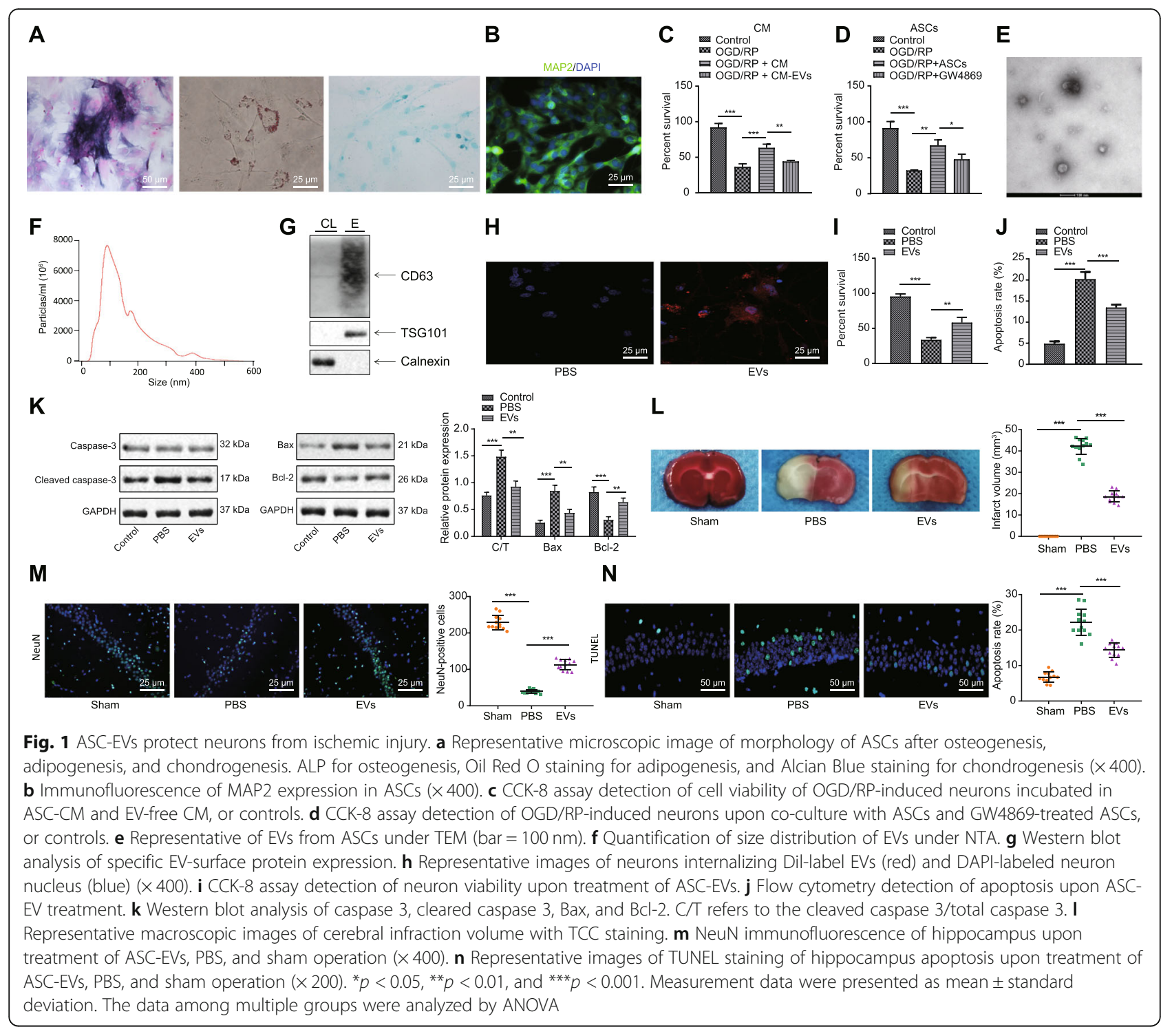

nucleus labeled with DAPI, the neurons were incubated with ASC-EVs for $24 \mathrm{~h}$. Fluorescence microscopy showed that Dil-labeled EVs (red) were clustered around the neuronal nucleus (blue), reflecting the process of ASC-EV internalization (Fig. 1h). Apart from these findings, ASC-EVs remarkably increased viability and decreased the apoptosis induced by OGD/RP (Fig. 1i, j). It is well known that caspase 3 is a prominent mediator of apoptosis [33], while Bax and Bcl-2 are apoptosis-related genes [34]. Thus, we analyzed the effects through determination of the expression of caspase $3, \mathrm{Bcl}-2$, and Bax. Results showed that cleaved caspase 3/caspase 3 and Bax expression were decreased and $\mathrm{Bcl}-2$ expression was increased (Fig. 1k). To investigate the in vivo effect of EVs, we established the MACO rat model and treated the rats with PBS or ASC-EVs for 3 days. The infarct volume and apoptosis were reduced and NeuN-positive cells were increased upon ASC-EV injection (Fig. 1l-n), thus indicating that ASC-EVs could alleviate the injury caused by OGD/RP.

\section{miR-22-3p derived from ASC-EVs could transfer to neurons}

Previous studies revealed that overexpressing miR-22-3p could alleviate the injury to neurons caused by $\mathrm{I} / \mathrm{R}$ injury [21]. To test the prediction that ASC-EVs might exert a protective activity by transferring miR-22-3p, we performed a series of assays. First, incubation with ASCEVs induced an increase of miR-22-3p expression in neurons and cerebral tissues (Fig. 2a, b). Besides, ASCs were transfected with FITC-labeled miR-22-3p mimic and their EVs were then isolated and used to incubate with neurons. Strong green fluorescence appeared in neurons incubated with ASC-EVs (Fig. 2c). Interestingly, 

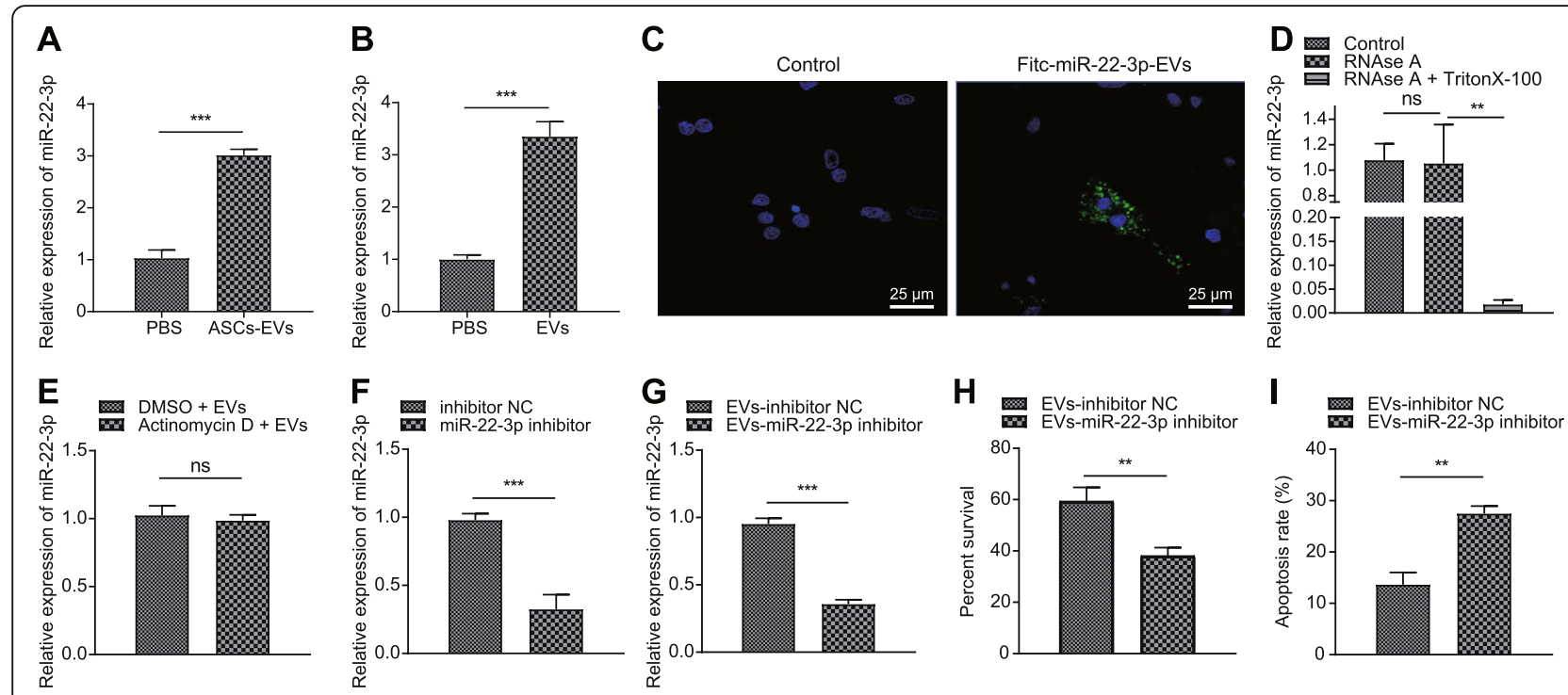

Fig. 2 ASC-EVs alleviate cerebral ischemic injury through miR-22-3p. a RT-qPCR analysis of miR-22-3p expression in neurons. $\mathbf{b}$ RT-qPCR analysis of miR-22-3p expression in cerebral tissues. $\mathbf{c}$ Representative microscopic images of FITC-miR-22-3p mimics in neurons ( $\times$ 400). $\mathbf{d}$ RT-qPCR analysis of miR-22-3p expression in neurons upon addition of RNase A in CM. e RT-qPCR analysis of miR-22-3p expression in neurons upon treatment of actinomycin D and ASC-EVs. $\mathbf{f}$ RT-qPCR analysis of miR-22-3p expression in ASCs upon miR-22-3p inhibitor. $\mathbf{g}$ RT-qPCR analysis of miR-22-3p expression in neurons incubated with the EVs from ASCs transfected with miR-22-3p inhibitor. $\mathbf{h}$ CCK-8 assay detection of neuron viability upon treatment of miR-22-3p inhibitor. i Flow cytometry detection of apoptosis upon treatment of miR-22-3p inhibitor. ${ }^{*} p<0.05$, ${ }^{* *} p<0.01$, and ${ }^{* *} p<0.001$. Measurement data were presented as mean \pm standard deviation. The data among multiple groups were analyzed by ANOVA

the addition of RNase A to CM of ASCs hardly altered miR-22-3p expression, but miR-22-3p could not be detected upon treatment with RNase A + Triton X-100 (Fig. 2d). The fact that actinomycin D did not alter miR22-3p expression excluded the possibility of endogenous induction (Fig. 2e), indicating that EVs or EV-derived miR-22-3p was internalized by neurons. Moreover, in the presence of the miR-22-3p inhibitor, apoptosis of neurons was increased, but their viability was decreased (Fig. 2f-i). Therefore, we conclude that ASC-EV-derived miR-22-3p (ASC-EV-miR-22-3p) could alleviate the response of neurons to ischemic injury.

\section{KDM6B is the target of miR-22-3p in neurons}

To further explore the downstream regulatory system of ASC-EV-miR-22-3p in cerebral ischemic injury, we searched the TargetScan database to predict downstream genes of miR-22-3p, which revealed a binding site of miR-22-3p at KDM6B 3'UTR 127-133 (Fig. 3a). Indeed, KDM6B has been shown to be highly expressed during cerebral ischemic injury [26]. Therefore, it is reasonable to suppose that miR-22-3p might attenuate cerebral ischemic injury by inhibiting the expression of KDM6B. To confirm the prediction from TargetScan, we performed the dual-luciferase reporter gene assay, which showed that co-transfection of KDM6B 3'UTR-WT and miR-22-3p mimic led to decreased luciferase activity. Nevertheless, no such difference appeared in KDM6B 3'UTR-MUT. These alterations of luciferase activity indicated that miR-22$3 p$ mimic specifically targeted at the 3 'UTR region of KDM6B (Fig. 3b). Besides, functional experiment indicated that transfection of miR-22-3p mimic led to a decrease in the mRNA and protein expression of KDM6B and that miR-22-3p inhibitor evoked the opposite effect (Fig. 3c-e). Moreover, when neurons were incubated with ASC-EVs, miR-22-3p expression was increased and KDM6B expression decreased (Fig. 3f-h). Collectively, ASC-EV-miR-22-3p could target and inhibit KDM6B expression in neurons.

\section{KDM6B promotes cerebral ischemic injury}

To investigate the impact of KDM6B on cerebral ischemic injury, three siRNAs against KDM6B and the plasmids overexpressing KDM6B were transfected into neurons. Using RT-qPCR and Western blot analysis, we confirmed the efficiency of oe-KDM6B and si-KDM6B as well as siRNAs, with siRNA2 exhibiting the most significant inhibitory effect (Fig. 4a, b). In the presence of OE-KDM6B, apoptosis was increased and viability was decreased in OGD/RP-induced neurons, as shown by CCK- 8 assay and flow cytometry (Fig. 4c, d). Moreover, cleaved caspase 3/caspase 3 and Bax expression were elevated, whereas $\mathrm{Bcl}-2$ declined (Fig. 4e). Collectively, the aforementioned evidence implies that KDM6B facilitated neuron apoptosis upon OGD/RP. 


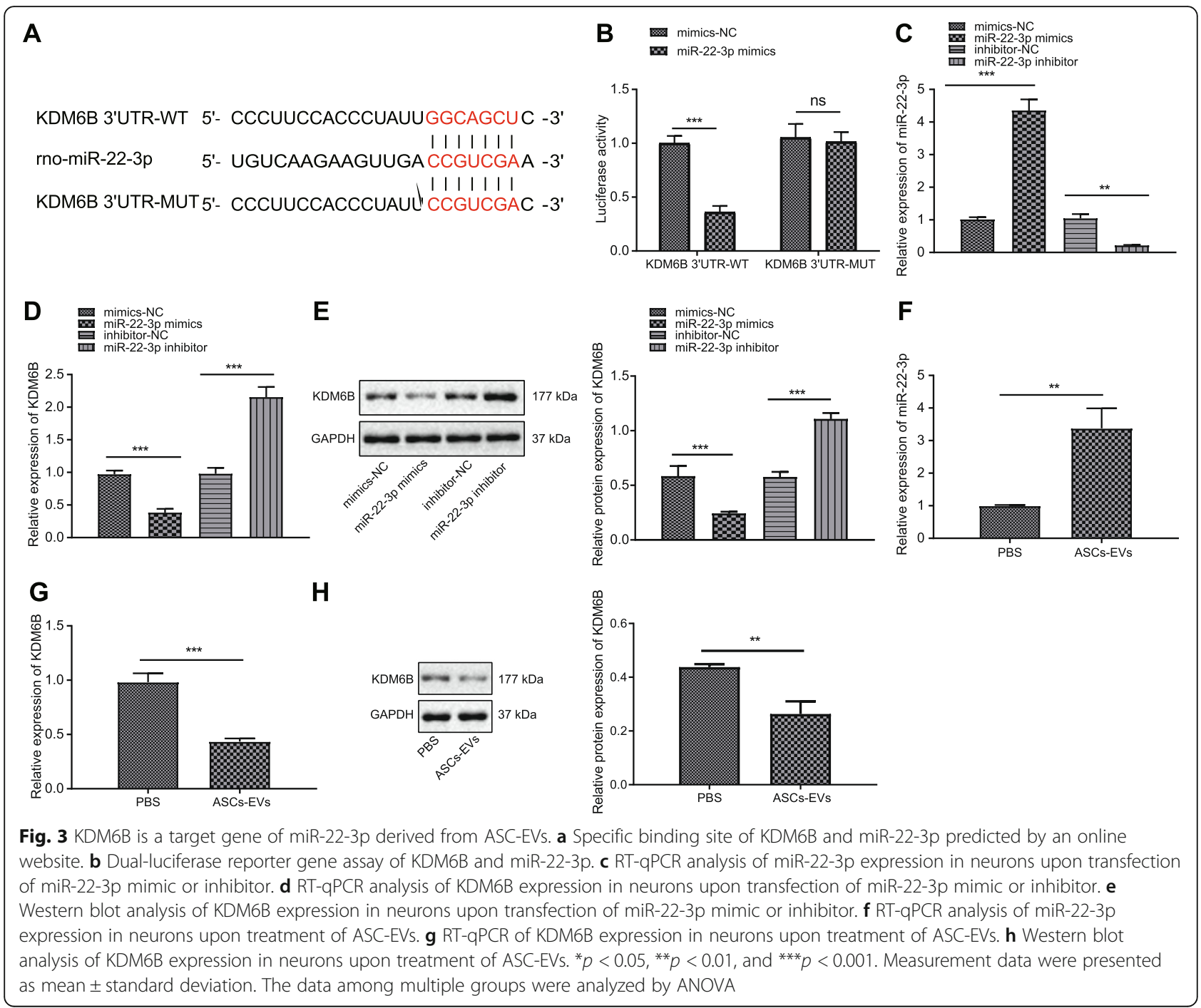

ASC-EVs protect neurons by inhibiting KDM6B expression To test the direct relation between ASC-EVs and KDM6B, neurons were transfected with OE-KDM6B and/or incubated with ASC-EVs. Results showed that treatment of ASC-EVs relative to PBS control remarkably increased miR-22-3p expression and decreased KDM6B expression in neurons, while OE-KDM6B + $\mathrm{PBS}$ relative to $\mathrm{OE}-\mathrm{NC}+\mathrm{PBS}$ increased KDM6B expression. Compared to the KDM6B level in ASC-EVs + OE$\mathrm{NC}$-treated neurons, the concentration was higher in ASC-EVs + OE-KDM6B-treated neurons (Fig. 5a-c). Besides, according to CCK- 8 assay and flow cytometry, OE-KDM6B treatment remarkably inhibited viability and enhanced apoptosis, while ASC-EV treatment had the opposite effect (Fig. 5d, e). OE-KDM5B + ASC-EVs treatment further inhibited apoptosis and increased viability. ASC-EV treatment clearly inhibited cleaved caspase 3, caspase 3, and Bax expression but increased Bcl2 expression, whereas treatment with OE-KDM6B had opposite effects (Fig. 5f). Taken together, we find that overexpression of KDM6B could reduce the protective effect of ASC-EVs on neurons.

\section{KDM6B promotes BMF expression via regulating BMP2}

Previous studies have noted that KDM6B could bind to BMP2 enhancer and promote BMP2 expression by depleting H3K27me3 [35]. Besides, BMP2 is implicated to be highly expressed in cerebral ischemic injury and could enhance BMF expression [36, 37]. Therefore, we next examined the possibility that KDM6B promotes ischemic injury by $\mathrm{BMP} 2 / \mathrm{BMF}$ regulation. Upon treatment of OE-KDM6B, BMP2 and BMF expression was increased, and conversely, si-KDM6B decreased the expression of BMP2 and BMP (Fig. 6a). Afterwards, we found that co-culturing neurons with ASC-EVs decreased the expression of KDM6B, BMP2, and BMP (Fig. 6b). Further, to check the interaction among KDM6B, BMP2, and BMP, we applied plasmids of OE- 


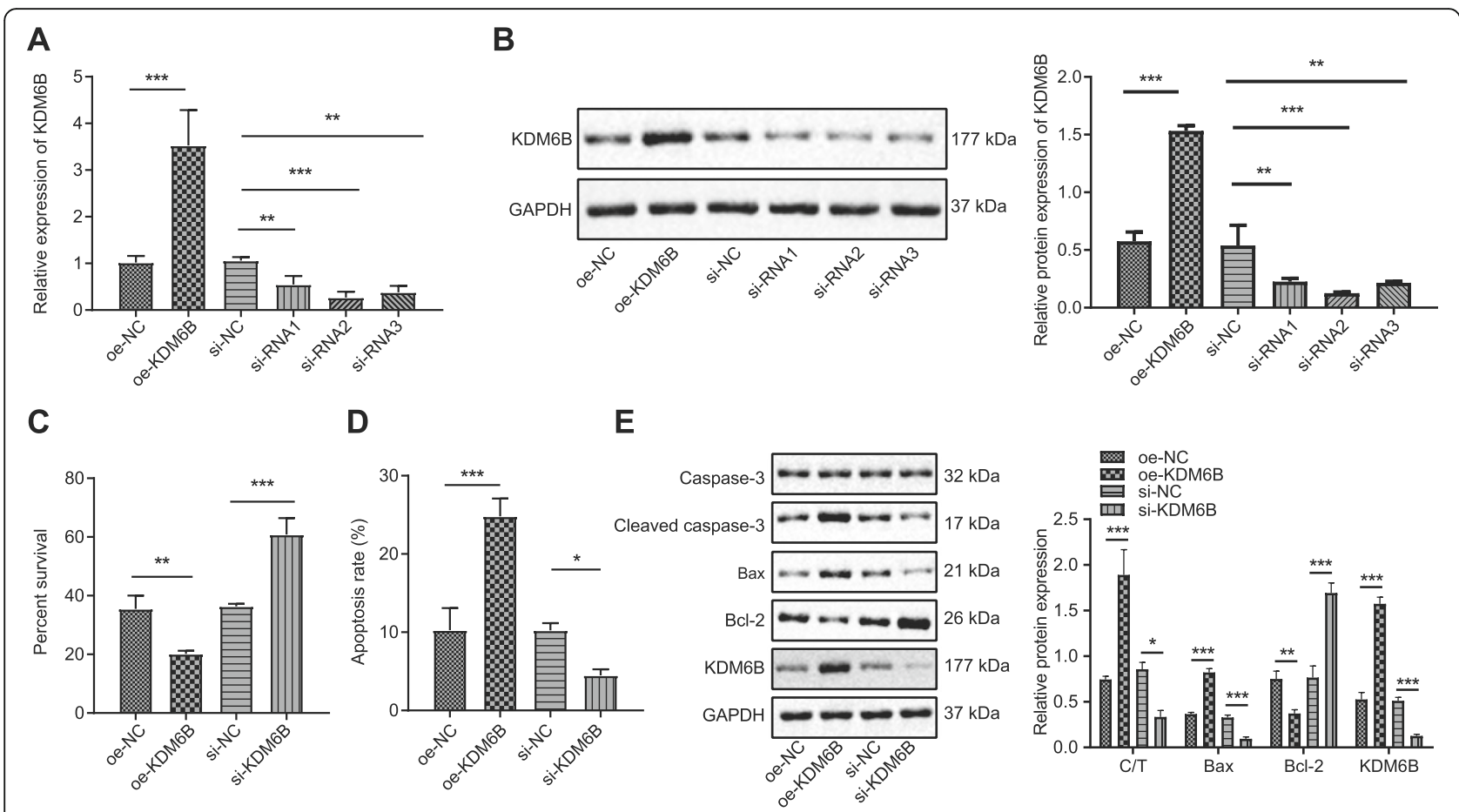

Fig. 4 High KDM6B expression exacerbates cerebral ischemic injury. a RT-qPCR analysis of KDM6B expression in neurons upon treatment of OEKDM6B or si-KDM6B. b Western blot analysis determining transfection effect of OE-KDM6B, siRNA1, siRNA2, siRNA3, and their corresponding controls. c CCK-8 assay detection of viability of neurons after treatment with OE-KDM6B or si-KDM6B, or controls. $\mathbf{d}$ Flow cytometry detection of apoptosis after treatment of OE-KDM6B and si-KDM6B, or controls. e Western blot analysis of cleaved caspase 3, caspase 3, Bax, and Bcl-2 protein expression after treatment of OE-KDM6B or si-KDM6B, or controls, and the corresponding quantification. ${ }^{*} p<0.05,{ }^{* *} p<0.01$, and ${ }^{* * *} p<0.001$. Measurement data were presented as mean \pm standard deviation. The data among multiple groups were analyzed by ANOVA

BMP2 and si-KDM6B, with confirmation by RT-qPCR of their inhibitory or mimicking activity in neurons (Fig. 6c). After neurons were simultaneously transfected with si-KDM6B and BMP2, Western blot analysis was conducted to detect the expression of KDM6B, BMP2, and BMF (Fig. 6d). Results showed that, compared to control treatment, $\mathrm{OE}-\mathrm{BMP} 2+\mathrm{si}-\mathrm{NC}$ treatment elevated the expression of BMP2 and BMF, while si-KDM6B + si$\mathrm{NC}$ treatment decreased the expression of KDM6B, $\mathrm{BMP} 2$, and BMF. Relative to si-KDM6B + si-NC treatment, the combination of si-KDM6B and OE-BMP2 increased the BMP2 and BMF levels, reversing the inhibitory effect of si-KDM6B on BMF. These data suggest that KDM6B increased BMF expression through mediating effects on BMP2 expression. To identify further this potential regulatory mechanism, we silenced KDM6B expression in neurons and carried out ChIP experiments to detect the binding between KDM6B and BMP2 promoter, also with H3K27me3 modification in the enhancer region (Fig. 6e). Results showed that siKDM6B remarkably inhibited the binding between KDM6B and the BMP2 promoter region but increased H3K27me3 modification in the enhancer region. Taken together, KDM6B depleted the H3K27me3 modification of BMP2 and promoted its expression, thereby enhancing BMF expression through binding to BMP2 enhancer region.

\section{ASC-EV-miR-22-3p alleviates cerebral ischemic injury through KDM6B-mediated BMP2/BMF axis}

To explore the mechanism whereby ASC-EV-miR-22-3p alleviates cerebral ischemic injury through BMF, we knock downed BMF expression in neurons through transfection with si-BMF. Since si-BMF1 exhibited the greatest inhibitory effect, this approach was selected for the following assays (Fig. 7a, b). Compared with EVinhibitor $\mathrm{NC}+$ si-NC, si-BMF + EV-inhibitor NC treatment reduced BMF expression, yet EV-miR-22-3p inhibitor + si-NC treatment promoted KDM6B, BMP2, and BMF expression (Fig. 7c, d). EV-miR-22-3p inhibitor and si-BMF treatment led to a decreased in BMF. Moreover, based on CCK-8 assay and flow cytometry, EVinhibitor $\mathrm{NC}+$ si-BMF treatment enhanced neuronal viability and suppressed apoptosis (Fig. 7e). EV-miR-22$3 p$ inhibitor treatment resulted in lower cell viability and increased apoptosis. However, the addition of si-BMF rescued the effect caused by EV-miR-22p inhibitor (Fig. 7f).

Animal experiments were also performed to test the in vivo effects of perturbing this mechanistic axis. After 


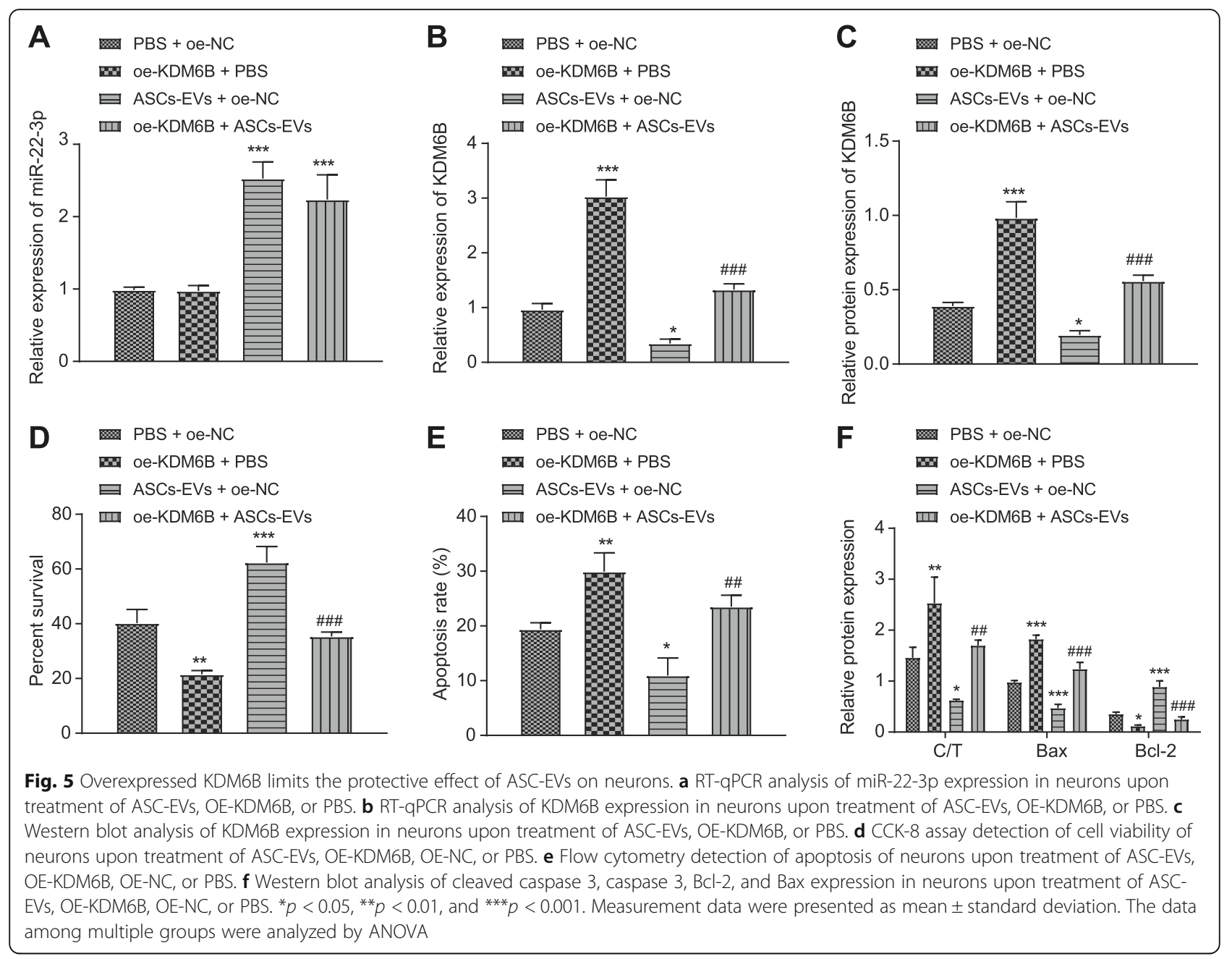

MACO, rats were injected with si-BMF or EVs, which showed that EV-miR-22-3p inhibitor led to increased infarcted volume, whereas the addition of si-BMF reduced the volume of infarct (Fig. 7g). As such, EV-miR-22-3p inhibitor enhanced apoptosis and reduced the number of $\mathrm{NeuN}^{+}$cells, which was reversed by the treatment of si-BMF (Fig. 7h, i). Therefore, we conclude that silencing BMF could alleviate ischemic injury. Taken altogether, ASC-EV-miR-22-3p alleviates cerebral ischemic injury by inhibiting the BMP2/BMF axis.

\section{Discussion}

Upon the onset of cerebral ischemic injury, oxygen and glucose are transiently depleted, inducing a cascade of deleterious cellular events including accumulation of excitatory glutamate in the extracellular space [38]. Normally, some limited spontaneous functional recovery takes place upon I/R injury [36]. Considering the limited treatment options for cerebral ischemic injury, new effective treatment strategies to protect the brain from I/R injury are urgently required [38]. Although recent years have witnessed the failure in the development of thousands neuroprotective drugs, neuroprotection based on novel pathways could still be attainable in stroke patients. In the present study, we demonstrated that ASCs alleviated the brain injury and reduced neuron apoptosis through EV-miR-22-3p.

MSCs can improve neuronal survival by promoting the anti-apoptotic signaling cascade, whereby paracrine factors secreted by MSCs protect neurons from apoptotic cell death in the OGD model of cerebral ischemia [39]. Besides, MSCs decreased OGD-induced apoptosis and inflammation after ischemic injury [40]. Systemic administration of MSC-EVs improved brain function by reducing seizures and preserving baroreceptor reflex sensitivity in a stroke model [12]. Even when administered after transient global cerebral ischemia, ASCs exhibited a prominent protective effect on neuron death [41]. Interestingly, auto-ASCs were more effective than allo-ASCs in reducing the infarct volume of MCAO rats [32]. Besides, ASC-CM could rescue normal axonal morphology, electrophysiological features, and cell 


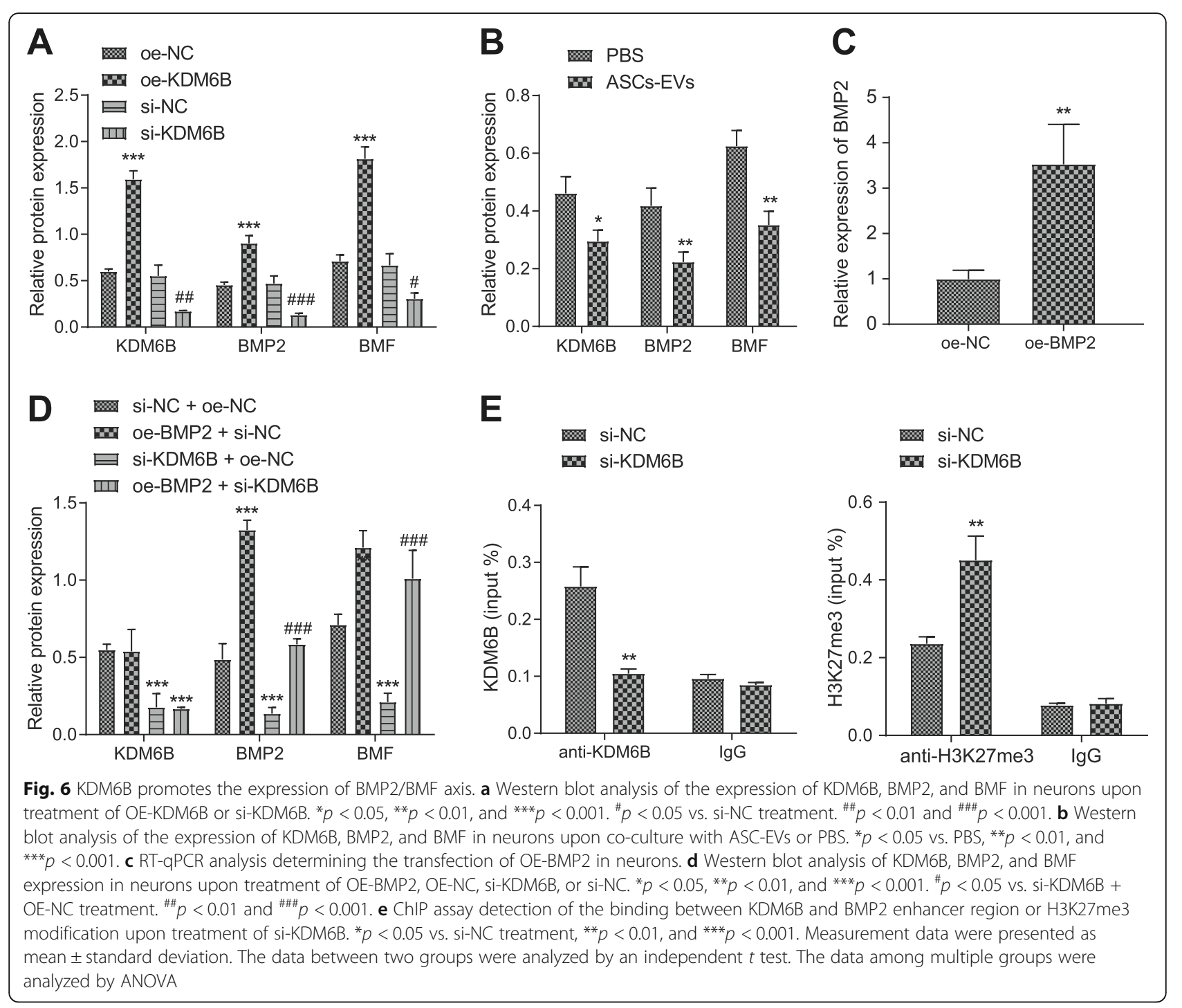

viability upon ischemic injury [42]. In the present study, the protective effect of ASCs on brain injury was confirmed and, moreover, ASC-CM with depletion of EVs was not effective as ASC-CM. This supported the potential role of EVs of ASCs in ischemic injury, which was further indicated by our finding that injection of EVs into MCAO rats significantly reduced the infarct volume. Likewise, MSC-EVs have been previously indicated to recover the impaired function and structural injury of brain upon hypoxia-ischemia [12]. Mechanistically, EVs induce longterm neuroprotection, neurological recovery, and favorable immune responses following ischemia [13].

Furthermore, our work unraveled that miR-22-3p from ASC-EVs attenuated apoptosis and brain injury by inhibiting KDM6B expression. Certain miRNAs such as miR-124 have been highlighted for their role in modulating signaling cascades to regulate stroke pathology, while these miRs promote or inhibit vascular endothelial cell biology and angiogenesis, which directly affects the progression of cerebral ischemia [43]. miRNAs from ASCEVs have been indicated to attenuate ischemic brain injury. For example, ASCs were shown to suppress inflammation and protect against brain injury by suppressing miR-21-3p, which directly inhibits protein expression [44]. In addition, miR-22-3p overexpression facilitated M2 polarization of macrophages and inhibited inflammation, thereby attenuating $I / R$ injury [45]. Moreover, caspase- 3 activity and Bax were inhibited by miR-22, and the expression of Bcl-2 in neurons was increased, such that miR-22 could protect against cerebral I/R injury [21]. Similarly, the current study also found that overexpressing miR-22-3p reduced the brain infarct volume, suppressed apoptosis, and enhanced viability of neurons. 


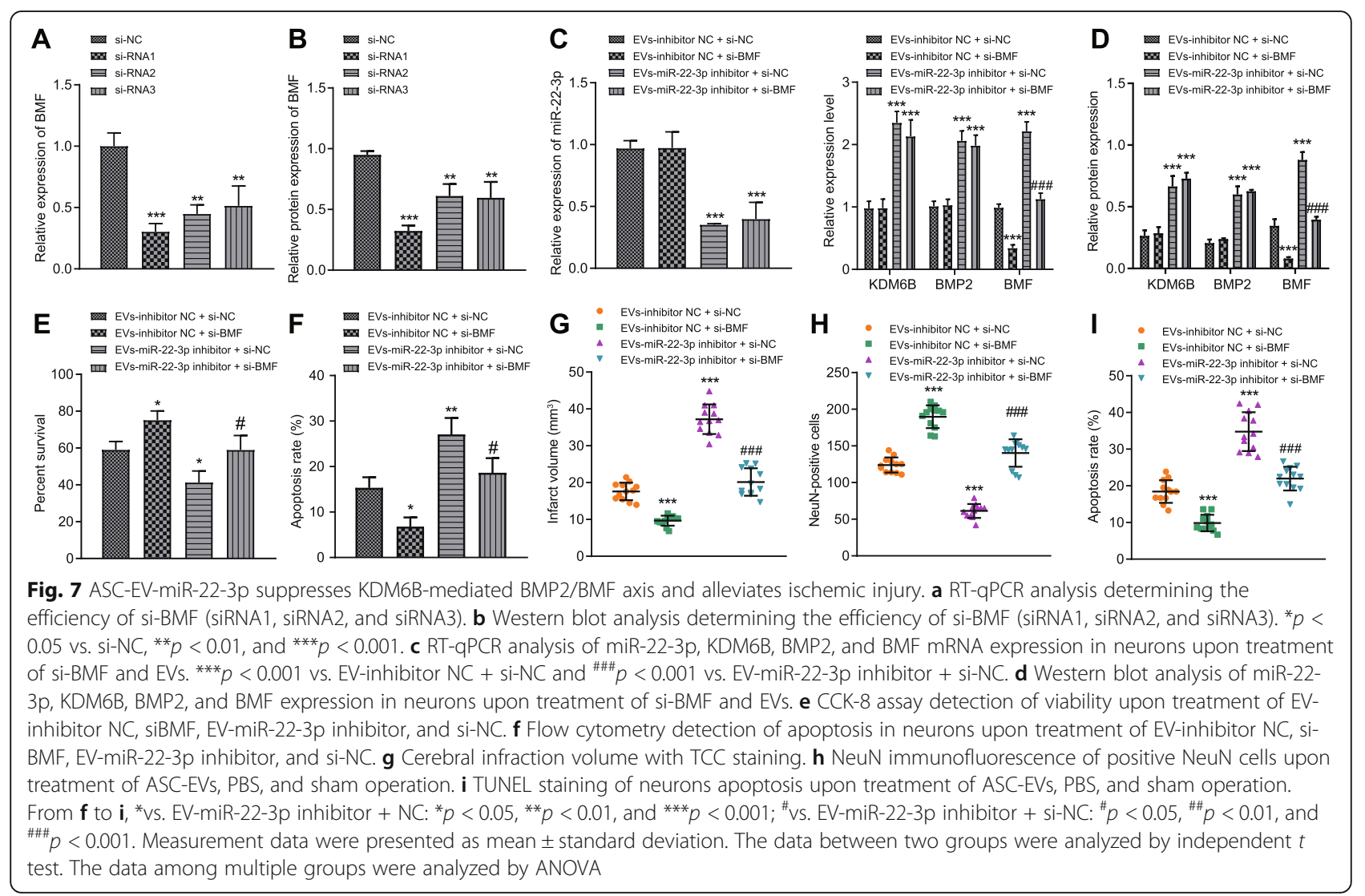

Cerebral I/R injury may cause neurological impairment in conjunction with caspase- 3 and Bax activation as well as Bcl-2 downregulation [46]. KDM6B (JMJD3) interacts with and recruits co-activators and transcription factors in the promoter region of the target genes to activate transcription [47]. JMJD3 is a critical promoter of neuronal apoptosis by regulating Bax as well as caspase-3, where silencing JMJD3 could improve neurological deficit and reduce the ischemic injury [26]. Consistent with these findings, our present results indicate that KDM6B worsened cerebral ischemic injury by activating caspase- 3 as well as Bax, while deactivating Bcl-2. Importantly, JMJD3 catalyzes the transition of H3K27me3 to H3K27me, thereby maintaining homeostasis by osteoblast differentiation, maturation, and apoptosis [48]. Mechanistically, KDM6B epigenetically activates neuronal genes by removing the repressive chromatin marker histone H3 lysine 27 trimethylation [49]. The present study unraveled that KDM6B promoted BMP2 expression by binding to the promoter region of BMP2 and depleting H3K27me3 modification. Similar to the present results, silencing KDM6B diminished the binding pattern of KDM6B in the BMP2 promoter regions in MSCs [27]. Under conditions of hypoxia, an increased level of H3K27me3 on the promoter region of BMP2, in combination with downregulation of KDM6B activity, suppressed osteogenic phenotypes of human periosteum-derived cells [50]. Another major finding of our study was that KDM6B promoted ischemic injury by enhancing BMF expression through BMP2. BMP2 is a neurotrophic factor, which induces the growth of midbrain dopaminergic neurons in vitro and in vivo, exerting neurotrophic effects [51]. Bone marrow MSCs increase BMP2/4 expression in ischemic astrocytes, enhancing subventricular progenitor cell gliogenesis by activating relevant signaling pathways and thus improving functional recovery after stroke [52]. Previous studies depicted that BMP2 expression was increased in the ischemic brain for as long as 4 weeks [36]. BMP-2 induces differentiation of ASCs into chondrocyte-like cells when promoting osteogenic gene expression levels and alkaline phosphatase activity in ASCs $[53,54]$. On the other hand, BMF provokes damage to the cytoskeleton and facilitates cytochrome formation [55]. In the presence of BMF overexpression, cell apoptosis was induced, whereas conversely, BMF knockdown protected neurons against death by incorporating with other important BH3-only proteins [56]. BMF overexpression causes neuron death and BMF knockdown protects neurons against death evoked by deprivation of nerve growth factor [56]. Moreover, silencing of BMF, in the present study, 


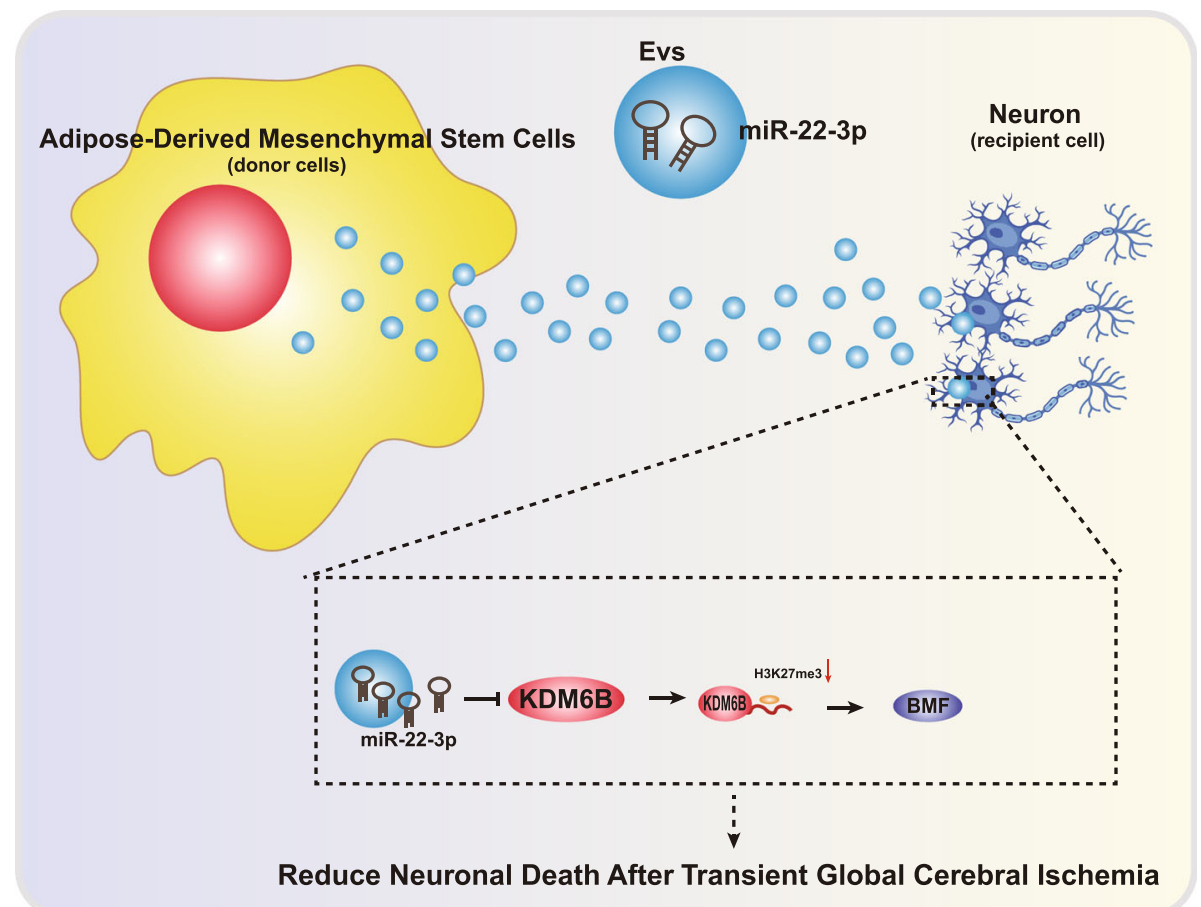

Fig. 8 Molecular schematic diagram concerning EV-miR-22-3p in cerebral ischemic injury. ASC-EVs could alleviate cerebral ischemic injury, where miR-22-3p was enriched in ASC-EVs. ASC-EV-miR-22-3p targeted and inhibited KDM6B expression, suppressing the binding between KDM6B and BMP2, and the decreased KDM6B downregulated BMF through the inhibition of BMP2 expression. Thereby, ASC-EV-miR-22-3p attenuates cerebral ischemic injury by suppressing KDM6B-mediated effects on the BMF/BMP2 axis

inhibited apoptosis induced by OGD/RP in vitro and reduced the volume brain lesions, by positively interacting with miR-22-3p.

\section{Conclusions}

Our work unraveled an underlying mechanism whereby ASCs could protect cerebral ischemic injury via EVmiR-22-3p by suppressing KDM6B-mediated effects on the BMF/BMP2 axis (Fig. 8). These findings could contribute to the development of a novel strategy of neuroprotection in cerebral ischemic injury.

\section{Abbreviations}

MCAO: Middle cerebral artery occlusion; OGD/RP: Oxygen-glucose deprivation/reperfusion; ASC-EVs: ASCs and ASC-derived EVs; MSC: Mesenchymal stem cell; EVs: Extracellular vesicles

\section{Acknowledgements}

We would like to give our sincere appreciation to the reviewers for their helpful comments on this article.

\section{Authors' contributions}

YMZ, JYL, MS, XW, and CCX designed the study. YMZ and JYL collated the data, carried out data analyses, and produced the initial draft of the manuscript. MS, XW, and CCX contributed to drafting the manuscript. All authors have read and approved the final submitted manuscript.

\section{Funding}

This study was supported by the project of Chengdu Science and Technology Bureau (2015-HM01-00157-SF), the Youth Fund Project of Chengdu University (2015XJZ11), the project of Sichuan Health Commission
(120580, 18PJ030, 20ZD018), Fund Project of Affiliated Hospital of Chengdu University (YZD2017005 and YP201503), and the project of Sichuan Education Department (17ZA0099).

\section{Availability of data and materials}

The datasets generated/analyzed during the current study are available.

\section{Ethics approval and consent to participate}

All animal experiments were performed with the approval of the Animal Ethics Committee of the Affiliated Hospital of Chengdu University. The experiments involving animals followed the recommendations in the Guide for the Care and Use of Laboratory Animals of the National Institutes of Health.

\section{Consent for publication}

Not applicable.

\section{Competing interests}

The authors declare no conflict of interest.

\section{Author details}

${ }^{1}$ Clinical Genetics Laboratory, Affiliated Hospital \& Clinical Medical College of Chengdu University, No. 82 North Second Section of Second Ring Road, Jinniu District, Chengdu 610081, Sichuan Province, People's Republic of China. ${ }^{2}$ Department of Neurology, Affiliated Hospital of Chengdu University, Chengdu 610081, People's Republic of China.

Received: 9 June 2020 Accepted: 9 December 2020

Published online: 05 February 2021

\section{References}

1. Campbell BCV, et al. Ischaemic stroke. Nat Rev Dis Primers. 2019;5(1):70.

2. Shi K, et al. Global brain inflammation in stroke. Lancet Neurol. 2019;18(11): 1058-66. 
3. Campbell BCV, Khatri P. Stroke Lancet. 2020;396(10244):129-42.

4. Humphris $\mathrm{JL}$, et al. Hypermutation in pancreatic cancer. Gastroenterology. 2017;152(1):68-74 e2.

5. Sommer CJ. Ischemic stroke: experimental models and reality. Acta Neuropathol. 2017;133(2):245-61.

6. Lee JA, et al. Mesenchymal stem-cell transplantation for hypoxic-ischemic brain injury in neonatal rat model. Pediatr Res. 2010;67(1):42-6.

7. Shiota Y, et al. Transplantation of a bone marrow mesenchymal stem cell line increases neuronal progenitor cell migration in a cerebral ischemia animal model. Sci Rep. 2018;8(1):14951.

8. Bonsack B, et al. Mesenchymal stem cell therapy alleviates the neuroinflammation associated with acquired brain injury. CNS Neurosci Ther. 2020;26(6):603-15.

9. Gutierrez-Fernandez $\mathrm{M}$, et al. Effects of intravenous administration of allogenic bone marrow- and adipose tissue-derived mesenchymal stem cells on functional recovery and brain repair markers in experimental ischemic stroke. Stem Cell Res Ther. 2013;4(1):11.

10. Chi $L$, et al. Tail vein infusion of adipose-derived mesenchymal stem cell alleviated inflammatory response and improved blood brain barrier condition by suppressing endoplasmic reticulum stress in a middle cerebral artery occlusion rat model. Med Sci Monit. 2018:24:3946-57.

11. van Niel G, D'Angelo G, Raposo G. Shedding light on the cell biology of extracellular vesicles. Nat Rev Mol Cell Biol. 2018;19(4):213-28.

12. Ophelders DR, et al. Mesenchymal stromal cell-derived extracellular vesicles protect the fetal brain after hypoxia-ischemia. Stem Cells Transl Med. 2016; 5(6):754-63

13. Doeppner TR, et al. Extracellular vesicles improve post-stroke neuroregeneration and prevent postischemic immunosuppression. Stem Cells Transl Med. 2015;4(10):1131-43.

14. Gowen A, et al. Mesenchymal stem cell-derived extracellular vesicles: challenges in clinical applications. Front Cell Dev Biol. 2020;8:149.

15. Jafarinia $\mathrm{M}$, et al. Therapeutic effects of extracellular vesicles from human adipose-derived mesenchymal stem cells on chronic experimental autoimmune encephalomyelitis. J Cell Physiol. 2020;https://doi.org/10.1002/ jср.29721.

16. Li T, et al. Adipose-derived mesenchymal stem cells and extracellular vesicles confer antitumor activity in preclinical treatment of breast cancer. Pharmacol Res. 2020;157:104843.

17. Serejo TRT, et al. Assessment of the immunosuppressive potential of INFgamma licensed adipose mesenchymal stem cells, their secretome and extracellular vesicles. Cells. 2019;8(1):22.

18. Huang $X$, et al. Exosomes derived from PEDF modified adipose-derived mesenchymal stem cells ameliorate cerebral ischemia-reperfusion injury by regulation of autophagy and apoptosis. Exp Cell Res. 2018;371(1):269-77.

19. Robbins PD, Dorronsoro A, Booker CN. Regulation of chronic inflammatory and immune processes by extracellular vesicles. J Clin Invest. 2016;126(4): 1173-80.

20. Lu TX, Rothenberg ME. MicroRNA. J Allergy Clin Immunol. 2018;141(4):12027.

21. Yu $H$, et al. Neuroprotective effects of viral overexpression of microRNA-22 in rat and cell models of cerebral ischemia-reperfusion injury. J Cell Biochem. 2015;116(2):233-41.

22. $L i B$, et al. miR-22-3p enhances the intrinsic regenerative abilities of primary sensory neurons via the CBL/p-EGFR/p-STAT3/GAP43/p-GAP43 axis. J Cell Physiol. 2020;235(5):4605-17.

23. Ye $L$, et al. Histone demethylases KDM4B and KDM6B promotes osteogenic differentiation of human MSCs. Cell Stem Cell. 2012;11(1):50-61.

24. Wei $Y$, et al. KDM6B overexpression activates innate immune signaling and impairs hematopoiesis in mice. Blood Adv. 2018;2(19):2491-504.

25. Guo Y, Xiong Z, Guo X. Histone demethylase KDM6B regulates human podocyte differentiation in vitro. Biochem J. 2019;476(12):1741-51.

26. Zhang $\mathrm{H}$, et al. Inhibiting Jumoji domain containing protein 3 (JMJD3) prevent neuronal apoptosis from stroke. Exp Neurol. 2018;308:132-42.

27. Naaijkens BA, et al. Human platelet lysate as a fetal bovine serum substitute improves human adipose-derived stromal cell culture for future cardiac repair applications. Cell Tissue Res. 2012;348(1):119-30.

28. Wang B, et al. A novel bacterial cellulose membrane immobilized with human umbilical cord mesenchymal stem cells-derived exosome prevents epidural fibrosis. Int J Nanomedicine. 2018;13:5257-73.

29. Verweij $F J$, et al. Analysis of viral microRNA exchange via exosomes in vitro and in vivo. Methods Mol Biol. 2013;1024:53-68.
30. Hawkins KE, et al. Embryonic stem cell-derived mesenchymal stem cells (MSCs) have a superior neuroprotective capacity over fetal MSCs in the hypoxic-ischemic mouse brain. Stem Cells Transl Med. 2018;7(5):439-49.

31. Leach MJ, et al. BW619C89, a glutamate release inhibitor, protects against focal cerebral ischemic damage. Stroke. 1993;24(7):1063-7.

32. Chung TN, et al. Adipose-derived mesenchymal stem cells reduce neuronal death after transient global cerebral ischemia through prevention of bloodbrain barrier disruption and endothelial damage. Stem Cells Transl Med. 2015;4(2):178-85.

33. Gu J, et al. Conserved function of Pacific cod Caspase-3 in apoptosis. Gene. 2020;732:144370.

34. Liu F, et al. Electroacupuncture ameliorates cognitive impairment and regulates the expression of apoptosis-related genes $\mathrm{BCl}-2$ and $\mathrm{Bax}$ in rats with cerebral ischaemia-reperfusion injury. Acupunct Med. 2015;33(6):47884.

35. Xu J, et al. KDM6B epigenetically regulates odontogenic differentiation of dental mesenchymal stem cells. Int J Oral Sci. 2013;5(4):200-5.

36. Shin JA, et al. Different temporal patterns in the expressions of bone morphogenetic proteins and noggin during astroglial scar formation after ischemic stroke. Cell Mol Neurobiol. 2012;32(4):587-97.

37. Larman BW, et al. Distinct bone morphogenetic proteins activate indistinguishable transcriptional responses in nephron epithelia including notch target genes. Cell Signal. 2012;24(1):257-64.

38. Huang L, Zhang L. Neural stem cell therapies and hypoxic-ischemic brain injury. Prog Neurobiol. 2019;173:1-17.

39. Scheibe F, et al. Mesenchymal stromal cells rescue cortical neurons from apoptotic cell death in an in vitro model of cerebral ischemia. Cell Mol Neurobiol. 2012;32(4):567-76.

40. Huang $P$, et al. Mechanism of mesenchymal stem cell-induced neuron recovery and anti-inflammation. Cytotherapy. 2014;16(10):1336-44.

41. Chung TN, et al. Effect of adipose-derived mesenchymal stem cell administration and mild hypothermia induction on delayed neuronal death after transient global cerebral ischemia. Crit Care Med. 2017;45(5):e508-e15.

42. Palomares T, et al. The neuroprotective effect of conditioned medium from human adipose-derived mesenchymal stem cells is impaired by N-acetyl cysteine supplementation. Mol Neurobiol. 2018;55(1):13-25.

43. Yin KJ, Hamblin M, Chen YE. Angiogenesis-regulating microRNAs and ischemic stroke. Curr Vasc Pharmacol. 2015;13(3):352-65.

44. Li C, et al. Adipose-derived mesenchymal stem cells attenuate ischemic brain injuries in rats by modulating miR-21-3p/MAT2B signaling transduction. Croat Med J. 2019;60(5):439-48.

45. Fang $\mathrm{H}$, et al. MicroRNA-22-3p alleviates spinal cord ischemia/reperfusion injury by modulating M2 macrophage polarization via IRF5. J Neurochem. 2020;https://doi.org/10.1111/jnc.15042.

46. Liu G, et al. Effects of apoptosis-related proteins caspase-3, Bax and BCl-2 on cerebral ischemia rats. Biomed Rep. 2013;1(6):861-7.

47. Zhang $X$, et al. JMJD3 in the regulation of human diseases. Protein Cell. 2019;10(12):864-82.

48. Yang $\mathrm{D}$, et al. The roles of histone demethylase Jmjd3 in osteoblast differentiation and apoptosis. J Clin Med. 2017;6(3):24.

49. Yang $\mathrm{L}$, et al. Histone demethylase KDM6B has an anti-tumorigenic function in neuroblastoma by promoting differentiation. Oncogenesis. 2019;8(1):3.

50. Yoon DK, et al. The involvement of histone methylation in osteoblastic differentiation of human periosteum-derived cells cultured in vitro under hypoxic conditions. Cell Biochem Funct. 2017;35(7):441-52.

51. Hegarty SV, Sullivan AM, O'Keeffe GW. Endocytosis contributes to BMP2induced Smad signalling and neuronal growth. Neurosci Lett. 2017;643:327.

52. Xin $\mathrm{H}$, et al. Bone marrow stromal cells induce BMP2/4 production in oxygen-glucose-deprived astrocytes, which promotes an astrocytic phenotype in adult subventricular progenitor cells. J Neurosci Res. 2006; 83(8): $1485-93$

53. An C, et al. IGF-1 and BMP-2 induces differentiation of adipose-derived mesenchymal stem cells into chondrocytes-like cells. Ann Biomed Eng. 2010;38(4):1647-54.

54. Lu Z, et al. Synergistic effect of nanomaterials and BMP-2 signalling in inducing osteogenic differentiation of adipose tissue-derived mesenchymal stem cells. Nanomedicine. 2015;11(1):219-28.

55. Hausmann M, et al. BCL-2 modifying factor (BMF) is a central regulator of anoikis in human intestinal epithelial cells. J Biol Chem. 2011;286(30):2653340. 
56. Akhter R, et al. The pro-apoptotic protein Bmf co-operates with Bim and Puma in neuron death induced by beta-amyloid or NGF deprivation. Mol Cell Neurosci. 2018;88:249-57.

\section{Publisher's Note}

Springer Nature remains neutral with regard to jurisdictional claims in published maps and institutional affiliations.

Ready to submit your research? Choose BMC and benefit from:

- fast, convenient online submission

- thorough peer review by experienced researchers in your field

- rapid publication on acceptance

- support for research data, including large and complex data types

- gold Open Access which fosters wider collaboration and increased citations

- maximum visibility for your research: over $100 \mathrm{M}$ website views per year

At $\mathrm{BMC}$, research is always in progress.

Learn more biomedcentral.com/submissions 